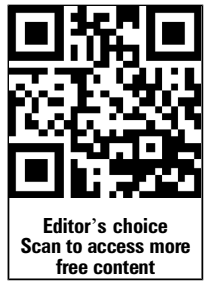

free content
- Additional material is published online only. To view please visit the journal online (http://dx.doi.org/10.1136/ thoraxjnl-2013-203998).

${ }^{1}$ Institute for Heart and Lung Health, Department of Medicine, The University of British Columbia, Vancouver British Columbia, Canada ${ }^{2}$ Collaboration for Outcomes Research and Evaluation, Faculty of Pharmaceutical Sciences, University of British Columbia, Vancouver, British Columbia, Canada

\section{Correspondence to}

Dr Mohsen Sadatsafavi, Centre for Clinical Epidemiology and Evaluation, 7th Floor, 828 West 10th Avenue, Research Pavilion, Vancouver, BC Canada V5Z 1M9; msafavi@mail.ubc.ca

Received 13 June 2013 Accepted 17 October 2013 Published Online First 27 November 2013

\title{
Dispensation of long-acting $\beta$ agonists with or without inhaled corticosteroids, and risk of asthma- related hospitalisation: a population-based study
}

\author{
Mohsen Sadatsafavi, ${ }^{1}$ Larry D Lynd, ${ }^{2}$ Carlo A Marra, ${ }^{2}$ J Mark FitzGerald ${ }^{1}$
}

\begin{abstract}
Background The role of long-acting $\beta$-agonists (LABA) added to inhaled corticosteroids (ICS) in the management of asthma is extensively debated. We thought to assess the risk of asthma-related hospitalisation in individuals who regularly filled prescriptions for ICS+LABA compared to those who regularly filled prescriptions for ICS alone or LABA alone, and compared to those who did not regularly fill such medications.
\end{abstract}

Methods Using administrative health databases of the province of British Columbia (BC), Canada, from 1997 to 2012, we conducted a nested case-control analysis of a cohort of asthma patients. Cases were defined as those who experienced asthma-related hospitalisation after the first year of their entry into the cohort. For each case, up to 20 controls were matched based on age, sex, date of cohort entry, and several measures of asthma severity. We categorised individuals as regularly exposed, irregularly exposed, or non-exposed to ICS alone, LABA alone, or ICS+LABA based on dispensation records in the past 12 months. The primary outcome measures were the rate ratio (RR) of the asthma-related hospitalisation among categories of regular exposure.

Results 3319 cases were matched to 43023 controls. The RR for regular dispensation of ICS+LABA was 1.14 (95\% Cl 0.93 to 1.41 ) compared with regular dispensation of ICS alone and $0.45(95 \% \mathrm{Cl} 0.29$ to 0.70) compared with regular dispensation of LABA alone. Those who regularly dispensed LABA had to dispense an ICS for at least three quarters of a year to reduce their risk to that of those who did not dispense LABA.

Conclusions Regular dispensation of ICS+LABA was not associated with an increased risk of asthma-related hospitalisation compared with regular dispensation of ICS alone. Adherence to ICS in patients who regularly receive ICS+LABA seems to be an important factor in the prevention of adverse asthma-related outcomes.

\section{INTRODUCTION}

The safety of long-acting $\beta$ agonists (LABA) in asthma has been the subject of much debate. ${ }^{1-4}$ While there is little doubt about the beneficial impact of LABA in reducing asthma symptoms, improving lung function and achieving clinical control, ${ }^{5-10}$ there is also compelling evidence that regular use of LABA as monotherapy increases the risk of serious asthma events, such as death and hospitalisations. ${ }^{411} 12$ Lingering concerns have led the US Food and Drug Administration (FDA) to

\section{Key messages}

What is the key question?

- Are asthma patients who regularly dispense inhaled steroids (ICS) and long-acting $\beta$ agonists (LABA) at increased risk of asthma-related hospitalisation compared with patients who regularly dispense ICS only?

What is the bottom line?

- This study did not show any difference in the risk between regular exposure to ICS+LABA versus ICS alone.

\section{Why read on?}

- Using administrative health data of an entire population of a well-defined geographic area, and by simultaneously contrasting the risk of asthma-related hospitalisation among individuals who regularly dispensed ICS and/or LABA, as well as those who did not regularly dispense any such medications, this study provides valuable information on the safety of LABA with and without concomitant ICS use in asthma.

require that manufacturers of LABA conduct randomised controlled trials (RCT) on the safety of the concomitant use of LABA and inhaled corticosteroids (ICS) versus ICS alone. ${ }^{1} 3$ The primary outcome of such trials is the composite event of asthma-related death, hospitalisation, or intubation, though it is already speculated that the results will be mainly driven by hospitalisation. ${ }^{3} 13$ The results of such RCTs will not be available for at least the next 5 years.

Health administrative databases provide a unique opportunity to examine the relation between drug exposure and rare outcomes. They provide large sample sizes and longitudinal information surpassing the follow-up time of feasible RCTs, and often consist of diverse and representative patient population. British Columbia (BC) is a western Canadian province with a population of 4.2 million, and a public health insurance system. The objective of this study was to use the comprehensive health administrative data of the province to evaluate the association between asthma-related hospitalisation and regular dispensation of ICS + LABA relative to regular dispensation of ICS alone or LABA alone among individuals with asthma. 


\section{METHODS}

This was a population-based nested case-control study of a cohort of patients who dispensed asthma medications identified using BC's provincial healthcare databases. ${ }^{14}$ We chose a nested case-control design because of the time-varying nature of the exposure, the complexities involved in ascertaining concomitant exposure to two medications, and the need for adjusting for temporal variations in the severity of asthma to overcome confounding by indication. The computational efficiencies of this design allowed us to contrast the risk of outcomes among several relevant exposure groups in a unified framework.

For the time period of April 1997 to March 2008, we had access to the databases recording basic demographic and socioeconomic variables, vital statistics, hospital discharge records (discharge diagnostic codes in either ICD-9th revision (ICD-9), or ICD-10), and outpatient medical services provided by practitioners (including one ICD-9 code for the service). We also had access to the PharmaNet, a database that captures information on all prescription drugs dispensed in the province, including a unique drug identifier, quantity and duration records. ${ }^{15}$ Additionally, once the study cohort was created, we could obtain updated data for individuals in the cohort till April 2012. All datasets are linkable at the individual level, and the data are shown to have very low rate of missing, underreporting, or misclassification. ${ }^{16-18}$ Since all legal residents of $\mathrm{BC}$ receive universal healthcare, the data are representative of the population. This database has successfully been used in addressing important drug safety questions. ${ }^{19-21}$

\section{Cohort definition}

Subjects were eligible for inclusion in the study if, during any 12-month window during which the subject's age was between 12 years and 45 years, they had filled at least three prescriptions for asthma-related medications (list available in the online supplementary material); however, once included, patients could remain in the cohort until they were 55 years old. ${ }^{22}$ For each patient, we defined the entry date as the date of the earliest dispensation of the first three dispensations that satisfied this criterion. In line with similar studies, ${ }^{22}$ we imposed a 12 -month baseline period, during which the asthma-related hospitalisations were not considered towards the outcome, to ensure that there were sufficient longitudinal data to measure the regularity of exposure, and to protect against the immortal time bias associated with the ascertainment of exposure over time. Therefore, the time zero for the analysis was 12 months after the entry date. All outcome dates were defined relative to time zero. Subjects were followed from time zero to the exit date defined as the last date of registration with the health system, the date the patient became 55 years, date of death, or $1 / 4 / 2012$, whichever came first.

\section{The outcome}

The main outcome of the study was asthma-related hospitalisation (the outcome). This was defined as a hospital discharge record with asthma (ICD-9:493.x, ICD-10: J45, J46) being the most responsible reason for hospitalisation. The hospital discharge database is maintained nationally by the Canadian Institute of Health Information, and undergoes regular validation studies (https://secure.cihi.ca/estore/productSeries.htm? $\mathrm{pc}=\mathrm{PCC} 228)$. A recent chart review of the data showed that the main diagnosis of asthma in a discharge record had a sensitivity of $87 \%$ and a positive predictive value of $90 \% .{ }^{17}$ Given that hospitalisations were included only after a case definition of asthma was satisfied, the likelihood of such hospitalisation being truly due to asthma should be even higher. Asthma-related deaths were not frequent enough to allow meaningful inference, and hence, were not evaluated as the outcome in the main analysis.

\section{Cases}

Within the cohort, we identified the first asthma-related hospitalisation occurring after time zero and before the exit date; the date of such hospitalisation relative to time zero was considered the index date.

\section{Controls}

We used incidence density sampling to construct a nested casecontrol dataset. ${ }^{23}$ For each hospitalisation, we first constructed a risk set consisting of all individuals who had been followed in the data at least as long as time-to-hospitalisation of their corresponding case. Each control was assigned an index date equal to the index date (relative to time zero) of the corresponding case. Controls could not have experienced asthma-related hospitalisation between their time zero and index date.

\section{Matching}

We matched controls with cases based on date of birth (within 2 years), date of entry (within 3 months), sex and six variables representing asthma severity, all previously used as proxies of asthma severity in observational studies. ${ }^{22} 2425$ For asthma severity variables, we dichotomised the value with the cut-off to be the midpoint between the average values among cases and controls. The resulting six binary matching variables were as follows: (1) any hospital admission due to asthma, (2) more than six dispensations for short-acting $\beta$ agonists (SABA); (3) any use of oral/parenteral corticosteroids; (4) any use of oral/ nebulised $\beta$ agonists; (5) more than five asthma-related dispensations other than steroids and $\beta$-agonists; and (6) more than four asthma-related outpatient services. All these variables were measured in the 12-month period before the index date, with the exception of asthma-related hospitalisation that was measured in the 12-month period after the entry date. For each case, up to 20 controls among the controls were selected at random.

\section{Exposure}

Based on drug dispensation records, each subject was categorised as being regularly exposed, irregularly exposed, or nonexposed to ICS alone, LABA alone, or ICS + LABA, henceforth referred to as study drugs, as follows: we divided the 12-month period immediately before the index date into four three-month periods and defined regular exposure to ICS +LABA as having at least one dispensation for ICS and one for LABA (or at least one dispensation for single-inhaler ICS+LABA) in each period. $^{22}$ Among individuals not satisfying the criterion for regular exposure to ICS+LABA, we defined regular exposure to ICS alone or regular exposure to LABA alone as having at least one record of dispensation for ICS (without any dispensation for LABA) or LABA (without any dispensation for ICS), respectively, during each period. Such a definition creates mutually exclusive categories for regular exposure to ICS alone, LABA alone and ICS +LABA, as well a 'no exposure' category not satisfying any regular exposure criteria. Additionally, subjects who did not satisfy the regular exposure criteria for a study drug, but had at least one dispensation for that drug in the year before the index date, were considered as irregularly exposed to that study drug. A similar method was used to further subclassify exposure to ICS+LABA in separate inhalers versus in a single inhaler. 
The latter group was further classified into exposure to budesonide + formeterol or fluticasone + salmeterol, the combination inhalers approved in Canada during the study period. In order to account for the potential stockpiling of drugs by patients, in above calculations we broke up dispensations with long days of supply (more than 30 days) to multiple consecutive dispensations.

\section{Analysis}

All analyses were performed in SAS V.9.3 (SAS Institute, Carry, North Carolina, USA). Two-tailed p values were considered significant at 0.05 . We compared the Rate Ratio (RR) of the outcome between cases and controls using conditional logistic regression for matched data. The distribution of baseline covariates and measures of asthma severity were presented for cases and controls, with appropriate weighting of controls for many-to-one matched data. ${ }^{26}$ The regression was adjusted for the exact values of the variables used for matching, as well as for socioeconomic status (represented by neighborhood-income quintiles) and several measures of comorbidity including non-asthma-related hospitalisations, non-asthma-related outpatient services, total number of medication dispensations, and the Charlson Comorbidity Index, ${ }^{27}$ all measured during the 12 months before the index date. The main regression included, aside from the covariates, variables representing regular and irregular use of the study medications. The reference group in this analysis, therefore, is individuals who did not fill prescription for any of the study medications. We also repeated the analysis with the reference group defined as those who did not fill or irregularly filled the study medication. This was performed by fitting the same regression model as above, but removing the variables representing irregular use.

The primary measures of interest were the pair-wise RRs of the outcome among those regularly exposed to ICS alone, LABA alone and ICS+LABA. Secondary outcomes were the RRs for regular exposure versus no exposure, and for regular exposure versus irregular/no exposure combined, for each study drug.

In order to study the impact of the regularity of exposure to ICS on the outcome among individuals who were regularly exposed to LABA, we created a variable counting the number of quarters in the 12-month period before the index date in which at least one ICS prescription was filled (a value between 0 and $4)$. We then measured the RR of the outcome across levels of this variable. A similar method was used to study the impact of the regularity of exposure to LABA among those who were regularly exposed to ICS. We performed several sensitivity analyses testing the impact of the different features of the design on the main results of the study, details of which are reported in the online supplementary material.

\section{RESULTS}

A total of 127081 individuals satisfied the cohort definition. The mean age at cohort entry was 28.1 (SD 10.3) years; and $63.8 \%$ were women. There was a total of 3319 first instances of hospitalisation after the 12-month baseline period, defining the case set for this analysis. These individuals were matched to 43023 controls (on average 13.0 controls per case).

Baseline demographic, asthma severity and comorbidity status of cases and controls are provided in table 1. Cases had indicators of more severe asthma than controls, even after matching, justifying further adjustment for such variables in the regression model. Cases also had higher measures of comorbidity than controls.

Overall, $4.4 \%, 12.2 \%$ and $0.5 \%$ of the cohort were classified as regularly exposed to ICS + LABA, ICS alone and LABA alone, respectively. Table 2 presents the cross-tabulation of case/control status and drug exposure groups.

The adjusted RRs for the outcome between exposure groups are presented in figure 1 . There was no statistically significant difference in the risk of the outcome among those who regularly filled prescriptions for ICS+LABA compared with those who

Table 1 Characteristics of cases and controls*

\begin{tabular}{|c|c|c|}
\hline & $\begin{array}{l}\text { Cases } \\
(\mathrm{n}=3319)\end{array}$ & $\begin{array}{l}\text { Controlst } \\
(n=43023)\end{array}$ \\
\hline $\begin{array}{l}\text { Age at entry (SD) } \\
\text { (median, (IQR)) }\end{array}$ & $\begin{array}{l}28.0(10.3) \\
(28.6(18.7-37.3))\end{array}$ & $\begin{array}{l}28.1(10.3) \\
(28.6(18.7-37.2))\end{array}$ \\
\hline $\begin{array}{l}\text { Age at index date (SD) } \\
\quad \text { (median, (IQR)) }\end{array}$ & $\begin{array}{l}32.6(11.0) \\
(33.6(23.3-42.1))\end{array}$ & $\begin{array}{l}32.6(11.0) \\
(33.5(23.2-42.1))\end{array}$ \\
\hline$\%$ Female & 63.8 & 63.8 \\
\hline \multicolumn{3}{|l|}{ Variables ascertained in the 12-month baseline period } \\
\hline Number of asthma-related hospitalisations in baseline & 0.20 & 0.05 \\
\hline \multicolumn{3}{|l|}{ Variables ascertained in the 12-month period prior to the index date } \\
\hline Number of dispensations of short-acting $\beta$ agonists & 7.34 & 6.43 \\
\hline Number of dispensations of oral or nebulised $\beta$ agonists & 0.48 & 0.31 \\
\hline Number of dispensations of oral or injected corticosteroids & 1.38 & 1.05 \\
\hline Number of asthma-related outpatient services§ & 8.18 & 5.45 \\
\hline Number of dispensations of other asthma-related medications & 9.73 & 8.49 \\
\hline Charlson comorbidity index & 0.96 & 0.85 \\
\hline Number of non-asthma-related hospitalisations & 1.15 & 1.07 \\
\hline Number of non-asthma-related outpatient services§ & 42.29 & 38.16 \\
\hline Number of dispensations of medications of all type & 26.31 & 20.78 \\
\hline
\end{tabular}


Table 2 Number and percentage of individuals by drug use type and regularity of use within cases and controls

\begin{tabular}{|c|c|c|c|c|c|c|}
\hline \multirow[b]{2}{*}{ Regular use of } & \multicolumn{3}{|l|}{$\begin{array}{l}\text { Cases, } n=3319 \\
n(\% \text { within row) }\end{array}$} & \multicolumn{3}{|l|}{$\begin{array}{l}\text { Controls, } n=43023 \\
n(\% \text { within row) }\end{array}$} \\
\hline & Regular exposure* & Irregular exposure & No exposuret & Regular exposure* & Irregular exposure & No exposure $†$ \\
\hline ICS+LABA $\ddagger$ & $180(5.4)$ & 585 (17.6) & $2554(77.0)$ & $1903(4.4)$ & $5088(11.8)$ & 36032 (83.8) \\
\hline Separate inhalers & $43(1.3)$ & $403(12.1)$ & $2873(86.6)$ & $436(1.0)$ & $1877(4.4)$ & 40710 (94.6) \\
\hline Single inhaler & $133(4.0)$ & $403(12.1)$ & $2783(83.9)$ & $1416(3.3)$ & $3320(7.7)$ & $38287(89.0)$ \\
\hline Budesonide+formoterol & $123(3.7)$ & $68(2.0)$ & $3128(94.2)$ & $1152(2.7)$ & $618(1.4)$ & $41253(95.9)$ \\
\hline Fluticasone+salmeterol & $84(2.5)$ & $290(8.7)$ & $2945(88.7)$ & $892(2.1)$ & $2294(5.3)$ & 39837 (92.6) \\
\hline ICS alone & $354(10.7)$ & $1483(44.7)$ & $1482(44.7)$ & 5259 (12.2) & 16676 (38.8) & $21088(49.0)$ \\
\hline LABA alone & $33(1.0)$ & $77(2.3)$ & 3209 (96.7) & $202(0.5)$ & $680(1.6)$ & 42141 (97.9) \\
\hline
\end{tabular}

* Regular exposure was defined as having a record of at least one dispensation of the corresponding medication in each of the four-month periods in the 12 months before the index date.

tNo exposure to ICS+LABA means the individual was not exposed to ICS nor to LABA in the 12 months prior to the index date. No exposure to ICS alone means the individual did not dispense ICS (either monotherapy or in a combined inhaler with LABA). Similarly, No exposure to LABA alone means the individual did not dispense LABA (either monotherapy or in a combined inhaler with ICS).

¥Number of regular ICS+LABA users is more than the sum of regular single-inhaler and regular double inhaler users because some individuals switched from single-inhaler to double-inhaler ICS+LABA, or vice versa, thus satisfying the criteria for regular ICS+LABA use but not the criteria for combination or separate inhaler use . The same reason causes the sum of irregular users of budesonide+formoterol and fluticasone+salmeterol to be more than the number of irregular users of single- inhaler ICS+LABA.

ICS, inhaled corticosteroids; LABA, long-acting $\beta$-agonists.

regularly filled prescriptions for ICS alone $(\mathrm{RR}=1.14(95 \% \mathrm{CI}$ 0.93 to 1.41$), \mathrm{p}=0.218)$. On the other hand, those who regularly filled prescriptions for ICS+LABA had a significantly reduced risk of the outcome compared to those who regularly dispensed LABA alone $(\mathrm{RR}=0.45 \quad(95 \% \mathrm{CI} 0.29$ to 0.70$)$, $\mathrm{p}<0.001)$. Similarly, regular dispensation of ICS alone was associated with a significantly lower risk of the outcome compared with regular dispensation of LABA alone $(\mathrm{RR}=0.40 \quad(95 \% \mathrm{CI}$ 0.26 to 0.60$), \mathrm{p}<0.001)$. No difference was detected between regular dispensation of ICS + LABA in separate inhalers versus in

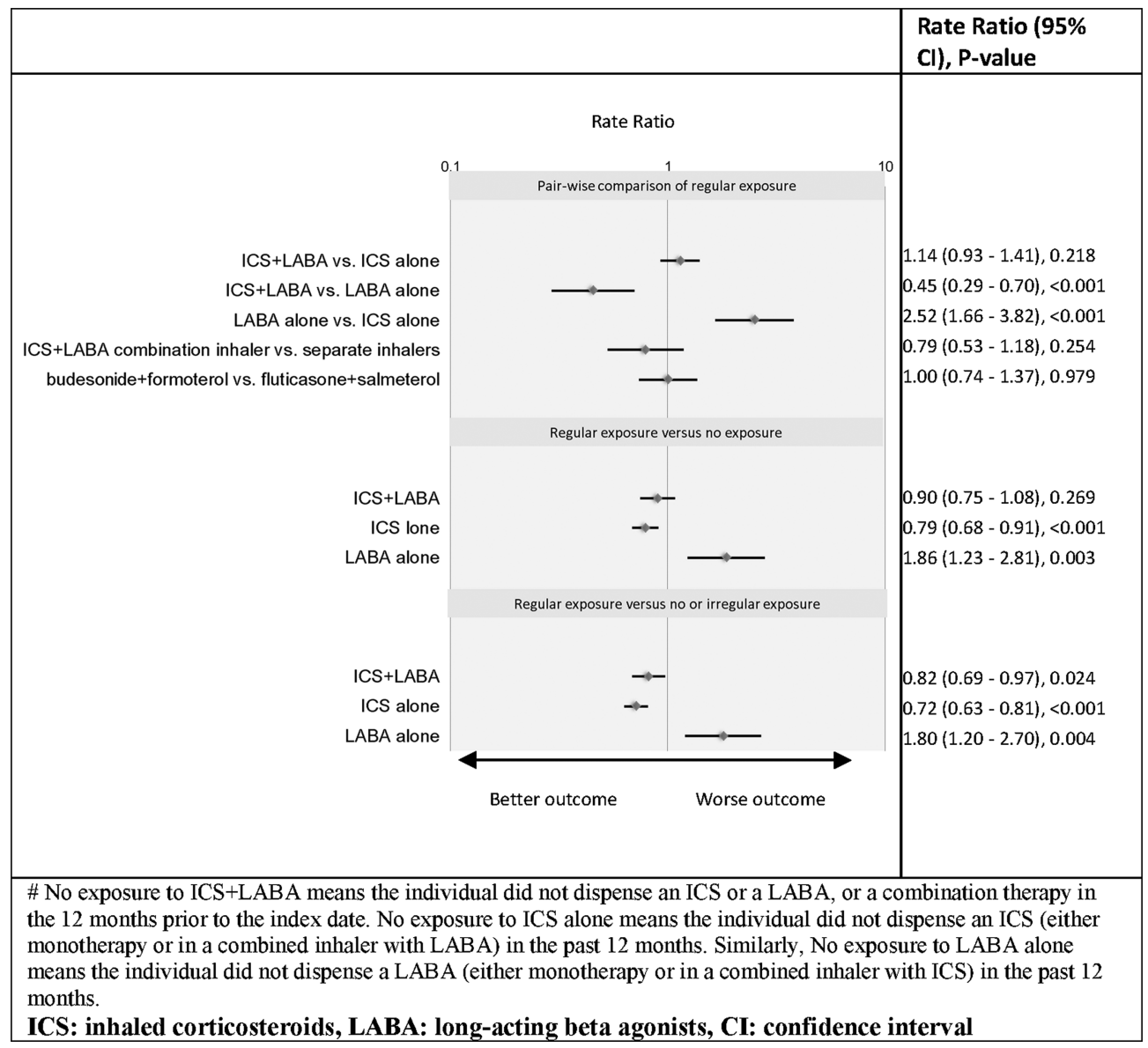

Figure 1 Forest plot of risk ratios (RR) of asthma-hospitalisation for exposure to inhaled corticosteroids (ICS)+ long-acting $\beta$-agonists (LABA), ICS, or LABA\#. 
a single inhaler, as well as between two different formulations of single-inhaler ICS+LABA (table 2 and figure 1).

When the reference group was defined as those who did not fill any prescription for ICS and/or LABA in the past year, regular dispensation of ICS + LABA was not associated with the outcome ( $\mathrm{RR}=0.90$ (95\% CI 0.75 to 1.08$), \mathrm{p}=0.269$ ); but regular dispensation of ICS was associated with a reduced risk of the outcome ( $R R=0.79$ (95\% CI 0.68 to 0.91$), p<0.001$ ). Those who regularly dispensed LABA alone, on the other hand, were at increased risk compared to this reference group $(\mathrm{RR}=1.86$ (95\% CI 1.23 to 2.81$), \mathrm{p}=0.003)$. When the reference group was defined as non-exposed/irregularly exposed combined, regular dispensation of ICS+LABA was associated with a significantly reduced risk of the outcome (figure 1).

Results of the analysis of the relation between ICS exposure and the outcome in those who regularly dispensed LABA are illustrated in figure 2. Among those who regularly dispensed LABA, each 3-month period with an ICS dispensation was associated with a $27.8 \%$ reduction in the risk of the outcome $(p=0.002)$. Among those who regularly dispensed ICS, the RR only minimally increased as a function of the number of quarters in which a LABA prescription was filled $(p=0.885)$.

Results of sensitivity analyses are presented in the online supplementary material. In all the analyses, the RR of ICS + LABA versus ICS remained non-significant (the CI included one), while regular dispensation of ICS + LABA remained significantly associated with a lower risk of the outcome compared with regular dispensation of LABA. In two scenarios, when the analysis did not control for SABA use, and when individuals with a history of asthma-related hospitalisations at baseline were removed, regular dispensation of ICS+LABA versus no dispensation of ICS or LABA became associated with a significantly reduced risk of the outcome.

\section{DISCUSSION}

We investigated the association between regular dispensation of ICS and/or LABA and the risk of hospitalisation due to asthma. According to our results, there was no significant difference in the risk among those who regularly dispensed ICS+LABA compared with individuals who regularly dispensed ICS alone. In none of the scenarios investigated in the sensitivity analysis was the risk associated with ICS+LABA significantly different than the risk associated with ICS alone, while there always remained a statistically significant reduced risk among those who regularly dispensed ICS + LABA compared with those who regularly dispensed LABA alone. Our results are also generally in line with previous findings that regular exposure to ICS reduces the risk of asthma-related hospitalisation, ${ }^{22}$ while regular exposure to LABA without concomitant ICS increases the rate of such events ${ }^{4}$ lending face validity to our overall findings.

Our analysis is based on 15 years of administrative health records of an entire population in a well-defined geographic area. This gives weight to the generalisability and applicability of research findings by reducing selection bias. The large sample size and the choice of the analytical framework allowed us to simultaneously contrast the risk of the outcome within several exposure groups, providing a broad picture on the interaction between exposure to ICS and/or LABA and the risk of asthma-related hospitalisation. We also believe the assessment of the regular exposure to ICS+LABA over a long-term period, as performed in the present study, is directly relevant to current policy concerns. There are, however, limitations to our study. Our case definition of asthma, although validated and used by other investigators, ${ }^{22}$ was based on medication records. The assessment of exposure was based on drug dispensation records and not drug intake. Patients who fill a prescription do not necessarily use the medication, or use it for the indented period. Additionally, some patients might receive drug samples from their physicians which are not captured in the health records. An important issue surrounding administrative data analysis for pharmaco-epidemiology studies is the potential for confounding by indication or disease severity. ${ }^{28}$ In the context of this study, this type of confounding may arise when patients who fill higher number of prescriptions may have more severe asthma, and thus, are at greater risk of experiencing adverse asthma-related outcomes. We tried to minimise such confounding by matching and adjustment by several measures of asthma severity. Residual confounding by indication is unlikely to be responsible for the observed results in this study; for such a confounding would have acted towards increasing the RR towards a harmful effect for the comparison of ICS + LABA versus monotherapy with ICS or with LABA.

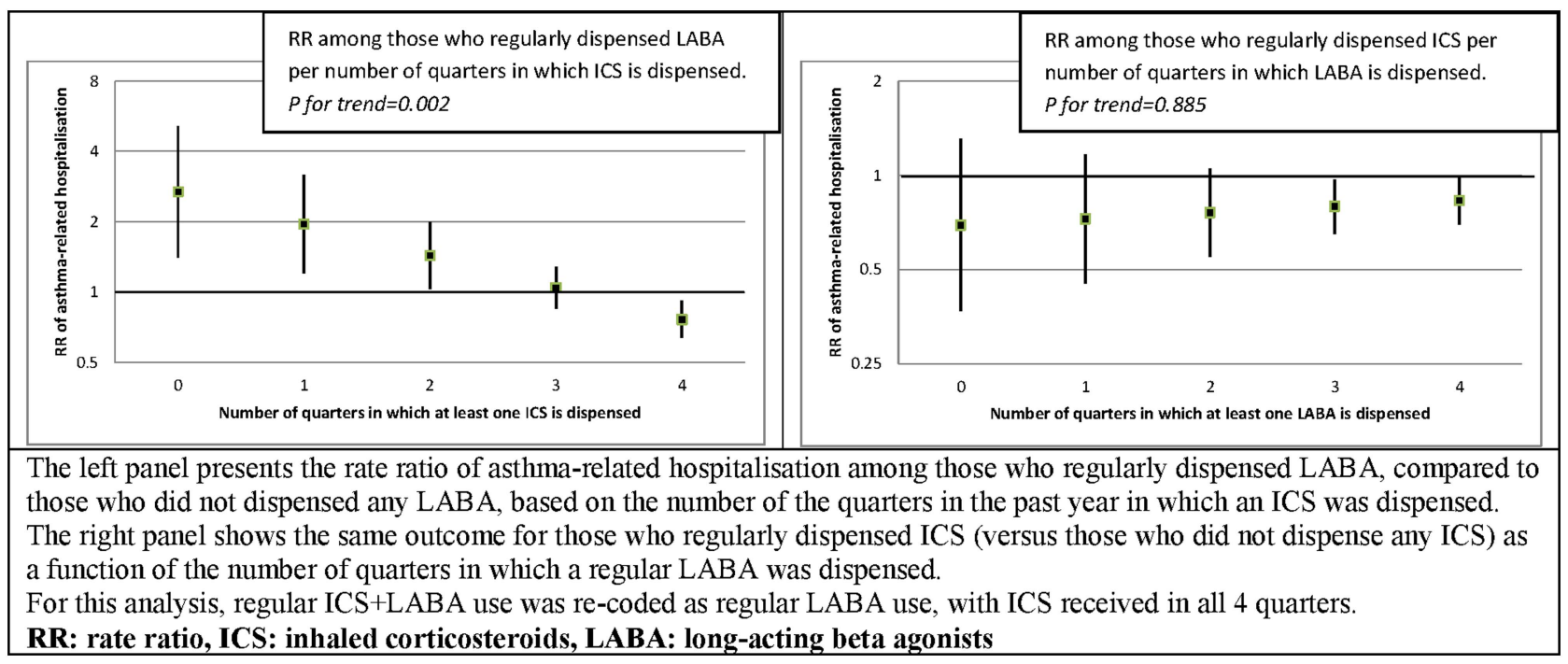

Figure 2 The impact of the regularity of inhaled corticosteroids (ICS) use in regular long-acting $\beta$-agonists (LABA) users, and vice versa. 
Other investigators have reported on the safety of ICS +LABA in asthma. ${ }^{29} 30$ In a case-control study involving 333 cases of asthma-related hospitalisations, Wang et $a l^{29}$ reported an adjusted OR for asthma-related hospitalisation of 0.54 for current exposure versus no exposure to ICS+LABA. The authors did not report the comparative safety of ICS+LABA versus ICS which is the centre of the debate and the outcome of the FDA-required trials. In a retrospective cohort study of the health records of 1828 individuals with asthma, Wells et al ${ }^{30}$ studied the relation between exposure to ICS+LABA or ICS alone in the previous 180 days and severe asthma exacerbations. They observed that the addition of LABA to ICS was associated with a significant reduction in the risk of the outcome $(p=0.049)$. Both the aforementioned studies were smaller studies based on US commercial health insurance data, thus, their generalisability is in doubt. The FDA's meta-analysis of LABA trials found a statistically significant increase in the risk of the composite event of asthma-related hospitalisation, intubation, or death in the trials in which users of LABA did not use ICS, whereas no significant change in risk was observed for ICS +LABA versus ICS. ${ }^{12}$ Our results are in line with these findings. On the basis of nearly 20 systematic reviews and database studies, Rodrigo et $a l^{4}$ reached similar conclusions.

Currently, FDA recommendations state that LABA should be used in conjunction with other controller medications for the shortest duration of time, and discontinued once asthma control is achieved. ${ }^{31}$ This poses challenges on how to stop LABA in patients who only achieve control with the addition of a LABA. The lack of association between regular exposure to ICS+LABA compared with ICS alone and the risk of hospitalisation in our study provides evidence that as long as adherence to ICS is ensured, there might be no need for such a step-off approach. This is especially relevant in the light of the recent evidence on the increase in asthma-associated impairment from the discontinuation of LABA. ${ }^{32}$

There is a large body of literature suggesting that if a patient's asthma remains uncontrolled despite regular use of ICS, the addition of a LABA provides superior asthma control than increasing the dose of ICS, ${ }^{33} 34$ but there is a lack of consensus on the safety of this therapy. The results of the trials required by the FDA will not be available for some years, and there are already concerns about the power of such RCTs. ${ }^{4}$ The observed RR of the outcome between ICS + LABA and ICS alone satisfies the non-inferiority criterion of such RCTs (the upper bound of CI being less than 2) by a wide margin. Even at the maximum increased risk $(\mathrm{RR}=1.41)$ compatible with the results of the present study, the trials will likely fail to detect any difference in the risk. ${ }^{13}$ In the meantime, the results of the present study, especially if confirmed by future research, provide evidence to patients, clinicians and policy makers with regard to the safety of combination therapy with ICS and LABA.

Correction notice This article has been corrected since it was published Online First. The word budenoside has been updated to read budesonide in figure 1 and table 2.

Contributors JMF conceived the research idea. JMF, MS, LDL and CAM devised the detailed study plan. MS performed the statistical analysis and wrote the first draft of the manuscript. All authors revised the manuscript and approved the final version for submission. Guarantor's name: Stirling Bryan.

Funding This study is part of the project 'Platform for Outcomes Research and Translation in Asthma and aLlergy (PORTAL)' funded by AllerGen National Center of Excellence.

Competing interests JMF has served on advisory boards for Glaxo-SmithKline, AstraZeneca, Novartis, Pfizer, Boehringer-Ingelheim, Altana, Merck and Topigen. He has also been a member of speakers' bureaus for Glaxo-SmithKline, AstraZeneca, Boehringer-Ingelheim, Pfizer and Merck. He has received research funding paid directly to the University of British Columbia from AstraZeneca, Glaxo-SmithKline, Boehringer-Ingelheim, Merck, Wyeth, Schering, Genentech and Topigen. He is a member of the Global Initiative for Asthma (GINA) and is chair of the GINA Science Committee. CAM has been a member of speakers' bureaus for Glaxo-SmithKline.

Ethics approval University of British Columbia Human Ethics Committee.

Provenance and peer review Not commissioned; externally peer reviewed.

Data sharing statement The provincial administrative data used in this study is owned by the Ministry of Health and, being considered personal information, is governed by the freedom of information and protection of privacy act (FIPPA). FIPPA not only prohibits the storage of Personal Information outside of Canada, but it also prohibits access, which includes viewing.

\section{REFERENCES}

1 Chowdhury BA, Dal Pan G. The FDA and safe use of long-acting beta-agonists in the treatment of asthma. N Engl J Med 2010;362:1169-71.

2 Kramer JM. Balancing the benefits and risks of inhaled long-acting beta-agoniststhe influence of values. N Eng/ J Med 2009;360:1592-5.

3 Chowdhury BA, Seymour SM, Levenson MS. Assessing the safety of adding LABAs to inhaled corticosteroids for treating asthma. N Engl J Med 2011;364:2473-5.

4 Rodrigo GJ, Castro-Rodríguez JA. Safety of long-acting \{beta\} agonists for the treatment of asthma: clearing the air. Thorax 2012;67:342-9.

5 Pearlman DS, Chervinsky P, LaForce C, et al. A comparison of salmeterol with albuterol in the treatment of mild-to-moderate asthma. $N$ Engl I Med 1992;327:1420-5.

6 Wenzel SE, Lumry W, Manning M, et al. Efficacy, safety, and effects on quality of life of salmeterol versus albuterol in patients with mild to moderate persistent asthma. Ann Allergy Asthma Immunol 1998:80:463-70.

7 Shrewsbury S, Pyke S, Britton M. Meta-analysis of increased dose of inhaled steroid or addition of salmeterol in symptomatic asthma (MIASMA). BMJ 2000;320:1368-73.

8 Greening AP, Ind PW, Northfield M, et al. Added salmeterol versus higher-dose corticosteroid in asthma patients with symptoms on existing inhaled corticosteroid. Allen \& Hanburys Limited UK Study Group. Lancet 1994;344:219-24.

9 Bateman ED, Boushey HA, Bousquet J, et al. Can quideline-defined asthma control be achieved? The Gaining Optimal Asthma ControL study. Am J Respir Crit Care Med 2004:170:836-44.

10 Salpeter SR, Buckley NS, Ormiston TM, et al. Meta-analysis: effect of long-acting beta-agonists on severe asthma exacerbations and asthma-related deaths. Ann Intern Med 2006;144:904-12.

11 Nelson HS, Weiss ST, Bleecker ER, et al. The Salmeterol Multicenter Asthma Research Trial: a comparison of usual pharmacotherapy for asthma or usual pharmacotherapy plus salmeterol. Chest 2006;129:15-26.

12 Levenson M. Long-acting beta-agonists and adverse asthma events meta-analysis. United States Food and Drug Administration, 2008.

13 Suissa S, Ariel A. US Food and Drug Administration-mandated trials of long-acting $\beta$-agonists safety in asthma: will we know the answer? Chest 2013;143:1208-13.

14 BC Ministry of Health. Population Data BC. http://www.popdata.bc.ca/data (accessed 20 Nov 2011).

15 Ministry of Health. PharmaNet. http://www.health.gov.bc.ca/pharmacare/pharmanet/ netindex.html (accessed 29 Mar 2012)

16 Williams J, Adger W. Inventory of studies on the accuracy of Canadian health administrative databases. Toronto: Institute for Clinical Evaluative Sciences, 1996.

17 Canadian Institute for Health Information. CIHI Data Quality Study of the 20052006 Discharge Abstract Database. Ottawa, Ontario, 2009. https://secure.cihi.cal free_products/DAD_DQ_study_2005_2006_August_2009_e.pdf (accessed 8 Sep 2012).

18 Hu W. Diagnostic Codes in MSP Claim Data, Summary Report. Victoria: Medical Services Plan, 1996

19 Solomon DH, Massarotti E, Garg R, et al. Association between disease-modifying antirheumatic drugs and diabetes risk in patients with rheumatoid arthritis and psoriasis. JAMA 2011;305:2525-31.

20 Schneeweiss S, Walker AM, Glynn RJ, et al. Outcomes of reference pricing for angiotensin-converting-enzyme inhibitors. N Engl J Med 2002;346:822-9.

21 Shirani A, Zhao Y, Karim ME, et al. Association between use of interferon beta and progression of disability in patients with relapsing-remitting multiple sclerosis. JAMA 2012;308:247-56

22 Suissa $\mathrm{S}$, Ernst $\mathrm{P}$, Kezouh A. Regular use of inhaled corticosteroids and the long term prevention of hospitalisation for asthma. Thorax 2002;57:880-4.

23 Mantel N. Synthetic retrospective studies and related topics. Biometrics 1973;29:479-86

24 Spitzer WO, Suissa S, Ernst $P$, et al. The use of beta-agonists and the risk of death and near death from asthma. N Engl J Med 1992;326:501-6.

25 Suissa $S$, Ernst $P$, Benayoun $S$, et al. Low-dose inhaled corticosteroids and the prevention of death from asthma. N Engl J Med 2000;343:332-6.

26 Schlesselman JJ, Stolley PD. Case-control studies: design, conduct, analysis. Oxford University Press, 1982 
27 Quan $\mathrm{H}$, Sundararajan V, Halfon P, et al. Coding algorithms for defining comorbidities in ICD-9-CM and ICD-10 administrative data. Med Care 2005;43:1130-9.

28 Hudson M, Suissa S. Avoiding common pitfalls in the analysis of observational studies of new treatments for rheumatoid arthritis. Arthritis Care Res (Hoboken) 2010;62:805-10

29 Wang M-T, Skrepnek GH, Armstrong E, et al. Use of salmeterol with and without concurrent use of inhaled corticosteroids and the risk of asthma-related hospitalization among patients with asthma. Curr Med Res Opin 2008;24:859-67.

30 Wells KE, Peterson EL, Ahmedani BK, et al. The relationship between combination inhaled corticosteroid and long-acting $\beta$-agonist use and severe asthma exacerbations in a diverse population. J Allergy Clin Immunol 2012;129: 1274-9.e2.
31 Postmarket Drug Safety Information for Patients and Providers. http://www.fda.gov/ Drugs/DrugSafety/PostmarketDrugSafetyInformationforPatientsandProviders/ ucm213836.htm (accessed 3 Apr 2012).

32 Brozek JL, Kraft M, Krishnan JA, et al. Long-acting $\beta 2$-agonist step-off in patients with controlled asthma: systematic review with meta-analysis. Arch Intern Med 2012;1-11. doi:10.1001/archinternmed.2012.3250

33 Gappa M, Zachgo W, von Berg A, et al. Add-on salmeterol compared to double dose fluticasone in pediatric asthma: a double-blind, randomized trial (VIAPAED). Pediatr Pulmonol 2009;44:1132-42.

34 Delea TE, Hagiwara M, Stanford RH, et al. Effects of fluticasone propionate/ salmeterol combination on asthma-related health care resource utilization and costs and adherence in children and adults with asthma. Clinical Therapeutics 2008;30:560-71. 